\title{
DIFFERENTIABLE OPEN MAPS ${ }^{1}$
}

\author{
BY P. T. CHURCH
}

Communicated by Deane Montgomery, June 4, 1962

Let $f: M^{n} \rightarrow N^{n}$ be a continuous function, where $M^{n}$ and $N^{n}$ are $n$-manifolds (without boundary). It will be implicitly assumed that the manifolds share the differentiability properties of $f$, e.g., $f \in C^{\prime}$ implies that $M^{n}$ and $N^{n}$ are $C^{\prime}$ manifolds. The map $f$ is called open if, whenever $U$ is open in $M^{n}, f(U)$ is open in $N^{n}$; it is light if, for every $y \in N^{n}, \operatorname{dim}\left(f^{-1}(y)\right) \leqq 0$.

For $n=2$, it is well-known that a nonconstant complex analytic function is open and light. Conversely, Stoilow proved that every light open map is locally, at each point, topologically equivalent $\left[9\right.$, p. 198] to one of the canonical analytic maps $g_{d}$, defined by $g_{d}(z)$ $=z^{d}(d=1,2, \cdots)$. If it is not assumed that $f$ is light, however, $f$ may be quite different from a $g_{d}$. R. D. Anderson in [1] (see also [2]) constructed an open map $f: S^{2} \rightarrow S^{2}$ such that, for each $y \in S^{2}$, $f^{-1}(y)$ is a nondegenerate continuum.

For $n \geqq 2$, let $F_{n, d}: E^{n} \rightarrow E^{n}$ be the canonical open map defined by: $F_{n, d}\left(x_{1}, x_{2}, \cdots, x_{n}\right)=\left(u_{1}, u_{2}, \cdots, u_{n}\right)$, where $u_{1}+i u_{2}$ $=\left(x_{1}+i x_{2}\right)^{d}(i=\sqrt{ }-1)$ and $u_{j}=x_{j}(j=3,4, \cdots, n ; d=1,2, \cdots)$. Since each $F_{n, d}$ is a generalization of $g_{d}$, it is natural to wonder (for $n \geqq 3$ ) how much an arbitrary open map $f$, satisfying some additional condition, differs locally from one of them.

The branch set $B_{f}$ is the set of points in $M^{n}$ at which $f$ fails to be a local homeomorphism (defined in [3]).

THEOREM. Let $f: M^{n} \rightarrow N^{n}$ be $C^{n}$ and open $(n \geqq 2)$, where $M^{n}$ is compact or $f$ is light. Then there exists a closed set $E, \operatorname{dim} E \leqq n-3$, such that, for each $x$ in $M^{n}-E$, there exists a neighborhood of $x$ on which $f$ is topologically equivalent to one of the canonical maps $F_{n, d}$ $(d=1,2, \cdots)$. Moreover, $E$ is nowhere dense in $B_{f}$ unless $f$ is a local homeomorphism.

In particular, for $n=2$ we have the classical structure. In [4, p. $620,(4.3)]$ there is a 2 -to- 1 open map $f: S^{5} \rightarrow S^{5}$ for which $B_{f}$ is not locally a manifold at any point (it is necessarily $[4$, p. $620,(4.2)]$ a $3-\mathrm{gm} \bmod 2)$; thus some differentiability assumption is required above. There is a $C^{\infty}$ open map $f: E^{2} \rightarrow E^{2}$ for which $B_{f}$ is the $y$-axis; thus either compactness of the domain or lightness of the map is needed. An example $f: E^{3} \rightarrow E^{3}$ (or $f: S^{3} \rightarrow S^{3}$ ) given by E. Hemmingsen

\footnotetext{
${ }^{1}$ Research supported in part by National Science Foundation grant 18049.
} 
and the author in $[4$, p. $620,(3.3)]$ indicates the extent of possible pathology. There $B_{f}$ has a Cantor set of point components, so that the exceptional set $E$ in the Theorem is necessary ( $f$ can be shown to be topologically equivalent to a $C^{\infty}$ map).

The following corollary is a generalization of the inverse function theorem. Let $Z$ be the set of zeros of the Jacobian determinant.

Corollary. If $f: E^{n} \rightarrow E^{n}, n \geqq 3, f \in C^{n}$, and $\operatorname{dim} Z \leqq 0$, then $f$ is a local homeomorphism.

Proof. The map $f$ is light, and its Jacobian determinant is either non-negative or nonpositive everywhere. Thus $f$ is open [8], and the result follows from the Theorem. More generally, the conclusion holds if $\operatorname{dim}\left(B_{f}\right) \leqq 0$.

A basic lemma for the proof of the Theorem follows. The set of points in $M^{n}$ at which the Jacobian matrix has rank at most $q$ is denoted by $R_{q}$.

Lemma. Let $h: M^{n} \rightarrow N^{p}$, where $h \in C^{n}$ and $M^{n}$ and $N^{p}$ are $n$ - and p-manifolds, respectively. Then $\operatorname{dim}\left(f\left(R_{q}\right)\right) \leqq q$.

In particular, $\operatorname{dim}\left(h\left(M^{n}\right)\right) \leqq n$. The lemma is related to the theorem of A. P. Morse [6] on the image of the critical set of a realvalued function, and to Sard's Theorem [7]. If $f$ is light, then [5, pp. 91-92] $\operatorname{dim}\left(R_{q}\right) \leqq q$.

The proof of the Theorem employs Morse's Theorem, a uniform form of the implicit function theorem, and some results from [3]. Detailed proofs will appear elsewhere.

\section{REFERENCES}

1. R. D. Anderson, On monotone interior mappings in the plane, Trans. Amer. Math. Soc. 73 (1952), 211-222.

2. - Open mappings of compact continua, Proc. Nat. Acad. Sci. U.S.A. 42 (1956), 347-349.

3. P. T. Church and E. Hemmingsen, Light open maps on n-manifolds, Duke Math. J. 27 (1960), 527-536.

4. - Light open maps on n-manifolds. II, Duke Math. J. 28 (1961), 607-624.

5. W. Hurewicz and H. Wallman, Dimension theory, Princeton Mathematical Series, Vol. 4, Princeton Univ. Press, Princeton, N. J., 1941.

6. A. P. Morse, The behavior of a function on its critical set of points, Ann. of Math. 40 (1939), 62-70.

7. A: Sard, The measure of the critical values of differentiable maps, Bull. Amer. Math. Soc. 48 (1942), 883-890.

8. C. J. Titus and G. S. Young, A Jacobian condition for interiority, Michigan Math. J. 1 (1952), 89-94.

9. G. T. Whyburn, Analytic topology, Amer. Math. Soc. Colloq. Publ. Vol. 28, Amer. Math. Soc., New York, 1942. 